\title{
Multiphoton absorption line shapes and branching ratios in intense laser fields: An application to $\mathrm{H}_{2}^{+}$photodissociation
}

\author{
Salvador Miret-Artés \\ Instituto de Física Fundamental, Consejo Superior de Investigaciones Científicas, \\ Serrano 123, 28006 Madrid, Spain \\ Osman Atabek \\ Laboratoire de Photophysique Moléculaire du Centre National de la Recherche Scientifique, \\ Université Paris-Sud Centre d'Orsay, 91405 Orsay, France \\ André D. Bandrauk \\ Département de Chimie, Faculté des Sciences, Université de Sherbrooke, Sherbrooke, Québec, Canada J1K 2R1 \\ (Received 24 October 1991)

\begin{abstract}
Time-dependent transition amplitudes between bound and continuum states coupled by an intense laser field are expressed in terms of their energy-dependent analogs maintaining the coherence effects through the intervention of complex molecular dressed states (field-induced resonances). A coupledequation method combined with an artificial-channel technique, properly describing the preparation step, is used to calculate absorption line shapes and branching ratios of the Floquet states involved in the multiphoton absorption-emission processes. The formalism is applied to the photodissociation of $\mathrm{H}_{2}{ }^{+}\left(1 s \sigma_{g}, v=0, j=1 \rightarrow 2 p \sigma_{u}\right)$. The intense-field behavior of laser-induced resonance Fano profiles are analyzed in relation with the dissociation line shapes. Strong mixings appear for intensities larger than $10^{13} \mathrm{~W} / \mathrm{cm}^{2}$ and the distribution of higher-energy peaks decreases with increasing intensity, due to stimulated emissions of the dissociating fragments.
\end{abstract}

PACS number(s): 42.50.Hz, 33.80.Gj, 33.80.Wz, 34.50.Rk

\section{INTRODUCTION}

It is now a well-known fact that multiphoton transitions in intense laser fields may radically change the dynamics of photodissociation. Nonlinear effects, abovethreshold absorption and dissociation leading to essential alterations in dynamical properties affecting optical spectra are expected for field intensities above $10^{11} \mathrm{~W} / \mathrm{cm}^{2}$. Such powerful lasers are currently operational and open new areas of interest, both experimental and theoretical, in problems of half collisions [1,2].

The theory of photodissociation puts the emphasis on different features according to the weak- or strong-field situations. The weak-field approach concentrates on complications inherent in the molecular dynamics. The photon mediates the preparation of the initial state and the absorption is described by calculating, through the Fermi golden rule, the cross section of a boundstate-continuum-state transition. This is the basis of the Franck-Condon principle which is a first-order perturbative approach. In the strong-field case, interest is rather focused on the description of the additional field-induced degrees of freedom (the molecule being generally assumed to be a simple diatomic). A model describing on an equal footing photon absorption, emission, and molecular dissociation should be of a nonperturbative nature and include the field in an appropriate way. In these treatments, the Fock (photon occupation number) representation is used which leads to dressed molecule or electronic-field surfaces involving discrete states embed- ded in continua to which they are radiatively coupled. The resulting laser-induced resonances monitor the fragmentation dynamics. A proper way to describe such a process relies on the calculation of resonance features (position and lifetime). Several methods are available, among them the solution of coupled equations for the multiphoton process, subject to Siegert-type boundary conditions [3].

In this context, it is important to emphasize that in the weak-field case the imaginary part of the quasienergy eigenvalue is directly related to the absorption cross section. But this is no longer valid in a strong-field regime, mainly due to the possible overlapping of resonances. In a previous work [3], we have shown the nonlinear behavior of the laser-induced resonances as a function of the field strength. But there is no one-to-one correspondence between a given resonance lifetime and the absorption cross section which is the only observable that can experimentally be reached. There remains the possibility to reconstruct the absorption line shape by combining the complete set of the resonances in a given energy region, but this may amount to the calculation of a very large number of quasienergies. A different, computationally attractive method is the time-independent full collisional treatment based on the introduction of two artificial channels as suggested by Bandrauk [4]. This treatment is a generalization of Shapiro's work [5], the first open artificial channel aiming to transform the otherwise halfcollision situation into a full collision and the second closed artificial channel being the true initial unperturbed 
(zero-field) molecular state, describing the preparation step. The power of the method relies on the fact that the summation over resonances can be carried out in an indirect way, the information being extracted from the scattering amplitude between the artificial entrance channel and the final physical continuum. As a consequence of the coupling scheme, the manifestation of the remaining resonances is through Fano's profiles. This paper is devoted to an application of the method to the case of $\mathrm{H}_{2}{ }^{+}$photodissociation to which a great deal of effort is directed both experimentally and theoretically [6,2,3].

The theory bridging the weak- and strong-field approaches is recalled in Sec. II. Results concerning the absorption line shape for several intensities as well as Fano's profiles and branching ratios describing different multiphoton processes are presented in Sec. III.

\section{THEORY}

A convenient method for calculating multiphoton cross sections, in intense-field dissociation, is the use of the $S$-matrix techniques in a rigorous time-dependent treatment. This may be done by a generalization of Shapiro's artificial-channel method for the calculation of direct photodissociation cross sections in weak-field situations. The total Hamiltonian for the molecule plus radiation field is taken in the following as:

$$
H=H_{m}+H_{\mathrm{rad}}+V \text {, }
$$

where $H_{m}$ and $H_{\text {rad }}$ are the isolated-molecule plus freeradiation parts, respectively. $V$ stands for the matterfield coupling. For the electronic-field (EF) gauge (socalled length form), we have

$V^{\mathrm{EF}}\left(\mathrm{cm}^{-1}\right)=1.17 \times 10^{-3} \mu($ a.u. $)\left[I\left(\mathrm{~W} / \mathrm{cm}^{2}\right)\right]^{1 / 2}$

$\mu$ being the electronic transition moment and $I$ the field intensity. Analogously, for the radiation-field (RF) gauge (velocity form), we have [3(b)]

$$
V^{\mathrm{RF}}=\left[\left(V_{c}-V_{a}\right) / \hbar \omega\right] V^{\mathrm{EF}}
$$

$V_{a}$ and $V_{c}$ being the bare potential energy surfaces of the initial, $|a\rangle$, and final, $|c\rangle$, electronic states, respectively, and $\omega$ the laser frequency. The unperturbed eigenstates of $H_{m}+H_{\text {rad }}$ are described by direct products of a molecular state (characterized by its electronic, vibrational, rotational quantum numbers summarized by the label $|a\rangle$ and energy $E_{a}$ ) and a field state (characterized by its pho- ton occupation number $|n\rangle$ and energy $n \hbar \omega)$, and symbolized by $|a, n\rangle=|a\rangle|n\rangle$,

$$
\left(H_{m}+H_{\mathrm{rad}}\right)|a\rangle|n\rangle=\left(E_{a}+n \hbar \omega\right)|a\rangle|n\rangle \text {. }
$$

For simplicity, the index $n$ will be usually dropped in this work.

The temporal evolution of some initial state $|\psi(0)\rangle$ is formally given by

$$
|\psi(t)\rangle=\exp (-i H t / \hbar)|\psi(0)\rangle
$$

Expanding in terms of eigenstates of $H_{m}+H_{\text {rad }}$ we can write Eq. (4) as

$$
|\psi(t)\rangle=\sum_{c^{\prime}} \int d E_{c^{\prime}}\left|c^{\prime}\right\rangle\left[\sum_{a^{\prime}} I_{c^{\prime} a^{\prime}}(t)\left\langle a^{\prime} \mid \psi(0)\right\rangle\right] .
$$

The discrete $c^{\prime}$ summation concerns all open continuum channels, belonging to either the ground-state or the excited-state manifolds with appropriate photon occupation numbers, whereas $a^{\prime}$ is an index for labeling the levels of the electronic ground state which are bound in field-free conditions. $I_{c^{\prime} a^{\prime}}(t)$ is the time-dependent transition amplitude between unperturbed bound $\left|a^{\prime}\right\rangle$ and energy-normalized continuum $\left|c^{\prime}\right\rangle$ states. Our aim is now to express this time-dependent amplitude in terms of matrix elements of the time-independent transition operator $T_{c^{\prime} a^{\prime}}$ which relate to the same states. We have to emphasize that, when describing intense-field multiphoton processes, one cannot refer to any perturbative expression such as the familiar Born expansion in terms of successive powers of the matter-field coupling, as the convergency may be questionable. If $I_{c^{\prime} a^{\prime}}(t)$ is given as a Fourier transform of the resolvent operator $G$

$$
I_{c^{\prime} a^{\prime}}(t)=(2 i \pi)^{-1} \int d E \exp (-i E t / \hbar) G_{c^{\prime} a^{\prime}}\left(E^{+}\right)
$$

and we use an integral relation between $G$ and $T$ [7]:

$$
G\left(E^{+}\right)=G_{0}\left(E^{+}\right)+G_{0}\left(E^{+}\right) T\left(E^{+}\right) G_{0}\left(E^{+}\right),
$$

where

$$
G\left(E^{+}\right)=\lim _{\varepsilon \rightarrow 0}(E+i \varepsilon-H)^{-1}
$$

and

$$
G_{0}\left(E^{+}\right)=\lim _{\varepsilon \rightarrow 0}\left(E+i \varepsilon-H_{m}-H_{\mathrm{rad}}\right)^{-1} .
$$

Equation (6) results into

$$
I_{c^{\prime} a^{\prime}}(t)=(2 i \pi)^{-1} \int d E \frac{T_{c^{\prime} a^{\prime}}(E) \exp (-i E t / \hbar)}{\left(E^{+}-E_{c^{\prime}}-n_{c^{\prime}} \hbar \omega\right)\left(E^{+}-E_{a^{\prime}}-n_{a^{\prime}} \hbar \omega\right)} .
$$

Upon complex integration, it can ultimately be shown that, when combining with Eq. (5), and referring to the limit of radiative lifetimes shorter than pulse lengths [4]:

$$
\lim _{t \rightarrow \infty}|\psi(t)\rangle=\sum_{c^{\prime}} \int d E_{c^{\prime}} T_{c^{\prime} a}\left(E_{c^{\prime}}\right) \frac{\exp \left[-i\left(E_{c^{\prime}}+n_{c^{\prime}} \hbar \omega\right) t / \hbar\right]}{\left[E_{c^{\prime}}+n_{c^{\prime}} \hbar \omega-E_{a}-n_{a} \hbar \omega\right]}\left|c^{\prime}\right\rangle
$$


provided the initial state $|\psi(0)\rangle$ is $|a\rangle$, i.e., one state of the electronic ground manifold. The total photodissociation probability $\boldsymbol{P}_{c a}$ for a transition from the initial bound state $|a\rangle$ to a continuum state $|c\rangle$ is then obtained by projecting $|\psi(t \rightarrow \infty)\rangle$ on the given $|c\rangle$ and integrating over all energies of this channel:

$$
P_{c a}=\int d E_{c}|\langle c \mid \psi(t \rightarrow \infty)\rangle|^{2} .
$$

The use of the orthogonality relation

$$
\left\langle c \mid c^{\prime}\right\rangle=\delta_{c c^{\prime}} \delta\left(E_{c}-E_{c^{\prime}}\right)
$$

cancels the discrete $c^{\prime}$ summation and the continuum $\int d E_{c^{\prime}}$ summation, and leads to

$$
P_{c a}(\omega)=\int d E_{c^{\prime}} \frac{\left|T_{c a}\left(E_{c^{\prime}}\right)\right|^{2}}{\left(E_{c^{\prime}}+n_{c^{\prime}} \hbar \omega-E_{a}-n_{a} \hbar \omega\right)^{2}} .
$$

A different presentation of the results is obtained by referring to branching ratios or partial widths of given final continua,

$$
\rho_{c a}(\omega)=\frac{P_{c a}(\omega)}{\sum_{c^{\prime}} P_{c^{\prime} a}(\omega)} .
$$

In the dressed-molecule picture limited to a singlephoton exchange that occurs in weak and intermediate fields where only zero- and one-photon states have to be taken into account. In strong fields, however, the situation is much more complicated due to the occurrence of cascades of absorption-emission processes. A set of dressed channels corresponding to different photon occupation numbers has to be defined which leads, through the Floquet Hamiltonian, and after integration over electronic and field variables, to the close-coupled equations written in compact form as

$$
\begin{aligned}
& {\left[H_{a a}+(n+1) \hbar \omega-E\right]|a, n+1\rangle} \\
& +V_{a c}(|c, n\rangle+|c, n+2\rangle)=0 \\
& \left(H_{c c}+n \hbar \omega-E\right)|c, n\rangle+V_{c a}(|a, n-1\rangle+|a, n+1\rangle)=0
\end{aligned}
$$

where $H_{a a}$ and $H_{c c}$ stand for the Hamiltonian of the uncoupled $|a\rangle$ and $|c\rangle$ states. These equations describe a half-collision process and may lead to the determination of the field-induced resonances as has been done recently [3]. The widths of these resonances are proportional to the photodissociation cross section when their mutual overlapping are considered as negligible. Such an assumption is not valid when strong fields are addressed. In that case, a possibility remains to transform artificially the process to a full collisional one, by adding an openentrance channel $\left|c_{1}\right\rangle$ as was first suggested by Shapiro [5]. $T_{c a}$ is then amenable to an indirect numerical evaluation via the element $S_{c c_{1}}$ of the scattering matrix.

As far as the weak-field limit is considered with a single photon absorbed, following Shapiro, the formalism can further be simplified by taking the couplings between $\left|c_{1}\right\rangle$ and $|a\rangle$ on one hand and $|a\rangle$ and $|c\rangle$ on the other hand as asymmetric $\left(V_{a c_{1}} \neq V_{c_{1} a}=0\right.$ and $V_{c a} \neq V_{a c}=0$, which means no emission). Then, no radiative shift and width affect the state $|a\rangle$ and only the second-order term of the Born expansion contributes to the $S$-matrix element,

$$
S_{c c_{1}}(E)=-2 i \pi \sum_{a} \frac{\langle c|V| a\rangle\left\langle a|V| c_{1}\right\rangle}{E-E_{a}-\hbar \omega} .
$$

It is then straightforward to show that for an energy $\widetilde{E}$ close enough to $E_{a}+\hbar \omega$, the sum over all states $|a\rangle$ cancels such that the residue of $S_{c c_{1}}$

$$
\langle c|V| a\rangle \simeq \frac{i}{2 \pi} \frac{\widetilde{E}-E_{a}-\hbar \omega}{\left\langle a|V| c_{1}\right\rangle} S_{c c_{1}}(\widetilde{E})
$$

is a first-order Born approximation to $T_{c a}$ calculated at the energy $\left(E_{a}+\hbar \omega\right)$. This leads to the well-known Fermi-golden-rule expression for the photofragmentation cross section from the initial $|a\rangle$ to the final $|c\rangle$ state due to the absorption of one photon of frequency $\omega$ :

$$
\sigma_{a}(\omega) \propto|\langle c|V| a\rangle|_{E_{a}+\hbar \omega}^{2} .
$$

Implicit in this derivation is that the initial state $|a\rangle$ is a well-defined, isolated, and unperturbed state. The photodissociation probability is calculated at the specific energy $E_{a}$ of state $|a\rangle$ dressed by the photon $\hbar \omega$. Clearly, this model may be extended to multiphoton transitions to a final continuum $\left|\psi_{c}^{(-)}\right\rangle$provided the first radiative transition from the state $|a\rangle$ is weak, without any further constraint concerning other couplings involved in the calculation of the incoming wave function $\left|\psi_{c}^{(-)}\right\rangle$conducted with physical symmetric radiative couplings ( $n$-photon simultaneous absorption-emission processes). Such a model has been used by Bandrauk and later by Shapiro to calculate the two-photon dissociation cross section $\mathrm{IBr}$ $[8,9]$.

Intense incident fields will however produce coherent mixing of the eigenstates of $H_{m}+H_{\text {rad }}$ via the radiative coupling $V$. Superposition of these states as well as the initially prepared state must be incorporated into the calculation. It is interesting to point out that by taking symmetric couplings for channels $|a\rangle$ and $|c\rangle\left(V_{c a}=V_{a c}\right.$, the artificial channel remaining asymmetrically coupled $\left.V_{a c_{1}} \neq V_{c_{1} a}=0\right)$ and using the operator relation

$$
T=V+T G_{0} V
$$

Eq. (16) leads to

$T_{c a}\left(E_{a}+\hbar \omega\right)=\frac{i}{2 \pi} \frac{1}{\left\langle a|V| c_{1}\right\rangle} R\left(S_{c c_{1}}\left(E_{a}+\hbar \omega\right)\right)$,

where $\mathcal{R}$ stands for the residue. This derivation does not involve any perturbative approximation for $T_{c a}$, as it is based upon the implicit relation given by Eq. (19). We note, however, that the cancellation of the sum over $|a\rangle$ gives $T_{c a}$ only at specific energies, i.e., that of the dressed unperturbed states $\left(E_{a}+\hbar \omega\right)$. Such energies have, contrary to the weak-field limit, no physical specificity since the field-induced resonances resulting from states $|a\rangle$ may be very much altered (shifted and broadened). The only meaningful information would be the total photodis- 
sociation probability given by Eq. (13) which necessitates the calculation of $T_{c a}$ at any energy. This is why the generalization of Eq. (16) may not be valid for very strongfield amplitudes. Bandrauk has recently proposed the introduction of a second bound artificial channel $|d\rangle$ which plays the role of the true initial unperturbed (zero-field) molecular state, weakly coupled to the total molecularfield manifolds [4]. This approach results in a simple numerical algorithm and has the merit of giving more insight into the dynamics by focusing on the field-induced resonances. In practice, $|d\rangle$ is some state $|a, n\rangle$, a condition which is achieved using an electronic potential $V_{d}$ identical to $V_{a}$ ensuring $\langle a \mid d\rangle=\delta_{a d}$ and $V_{a d}$ $=\langle a|V| d\rangle=1$ (weak coupling), whereas $V_{d a}=0$ and $V_{a c}=V_{c a} \neq 0$ (symmetric strong coupling). The two artificial channels $\left|c_{1}\right\rangle$ and $|d\rangle$ are supposed to be coupled in an asymmetric way, i.e., $V_{d c_{1}} \neq V_{c_{1} d}=0$ so as not to perturb the initial state $|d\rangle$ (no shift or width). Use of the formalism of projection operators leads, after some algebra, to

$S_{c c_{1}}(E)=-2 i \pi \sum_{d} \frac{\langle c|T| a\rangle\langle a|V| d\rangle\left\langle d|V| c_{1}\right\rangle}{\left(E-E_{a}-n_{a} \hbar \omega\right)\left(E-E_{d}\right)}$,

where

$$
\begin{aligned}
T_{c a}(E) & =\langle c|T| a\rangle \\
& =\left(E-E_{a}-n_{a} \hbar \omega\right) \sum_{L} \frac{\langle c|t| L\rangle\langle L \mid a\rangle}{\left(E-E_{L}+i \Gamma_{L} / 2\right)}
\end{aligned}
$$

with $t=V+V P(E-P H P)^{-1} P V, P$ being the projection operator over all continuum states. $|L\rangle$ corresponds to field-induced resonances with complex energies $E_{L}$ $-i \Gamma_{L} / 2$. More precisely, they are eigenstates of $H_{m}+H_{\mathrm{rad}}+Q t Q, Q$ being the projection operator over the discrete states. The overlaps $\langle L \mid a\rangle$ describe the preparation of the initial state $|a\rangle$ into the complex dressed states $|L\rangle$, as would occur in sudden excitation. The transition operator $t$ connects the dressed states $|L\rangle$ to the physical final continuum state $|c\rangle$. The coherenece effects of the intense field is thus maintained through the $|L\rangle$ states. Invoking the weak-coupling limit, one has $|L\rangle \rightarrow|a\rangle, \Gamma_{L} \rightarrow 0$, and $E_{L} \rightarrow E_{a}+n_{a} \hbar \omega$ so that one obtains with $t=V$

$$
T_{c a}(E)=\langle c|V| a\rangle
$$

according to weak-field conditions.

Concerning multiphoton dissociation, $T_{c a}$ can, in principle, be obtained by the sum of the contributions of all resonances as indicated by Eq. (22). Such a calculation is currently under investigation in our group. But it is also obtainable by the numerical evaluation of the $S$-matrix element, $S_{c c_{1}}$, from Eq. (21). The sum over $|d\rangle$ can be canceled by the choice of an energy $E$ close enough to $E_{d}$, but as this last energy can be varied at will, $T_{c a}(E)$ is obtained at any energy, such that Eq. (13) can now give the total photodissociation probability for a given final continuum $|c\rangle$. Here, again, we emphasize the nonperturbative nature of this treatment for calculating $T_{c a}$.

\section{RESULTS}

The theory which is presented in the preceding section is illustrated by the example of the multiphoton photodissociation of $\mathrm{H}_{2}{ }^{+}$bound in its electronic ground state

$$
\mathrm{H}_{2}{ }^{+}\left(1 s \sigma_{g}, v=0, j=1\right)+n \hbar \omega \rightarrow \mathrm{H}^{+}+\mathrm{H}(1 s)+\epsilon(n) .
$$

Figure 1 displays the collection of dressed channels (closed and open) described by the model. We only consider dissociation via the repulsive $2 p \sigma_{u}\left(\mathrm{H}_{2}{ }^{+}\right)$electronic state which is asymptotically degenerate with $1 s \sigma_{g}\left(\mathrm{H}_{2}{ }^{+}\right)$. The corresponding potential energy curves are given by Morse-type representations. Any change in the rotation quantum number due to the interaction with the field is neglected. The calculations, done in the velocity gauge, Eq. (2b), are presented by considering three intensity regimes for the electromagnetic field (weak, if the Rabi frequency $\hbar^{-1} V_{c a}^{\mathrm{EF}}$ is less than the vibrational frequency $\omega_{v}$ of $\mathrm{H}_{2}{ }^{+}$; intermediate if the two frequencies are comparable, strong if the Rabi frequency is larger).

\section{A. Weak-field regime}

As has been pointed out previously [3], this regime (valid for an intensity lower than $10^{11} \mathrm{~W} / \mathrm{cm}^{2}$ ) corresponds to the linear behavior of the photodissociation rate $\left|T_{c a}\right|^{2}$ versus the intensity. This also corresponds to the region where the Fermi golden rule is valid, such as a lowest-order perturbation formula, Eq. (18), can successfully be applied. We have performed a series of calculations for a laser wavelength varying from 60 to $200 \mathrm{~nm}$ and intensities ranging from $3.5 \times 10^{6}$ to $3.5 \times 10^{10}$ $\mathrm{W} / \mathrm{cm}^{2}$ by using the three techniques we have at our disposal, i.e., Fermi golden rule, Eq. (18), Shapiro's one artificial channel, Eq. (16), and the two-artificial-channel model, Eq. (21), by retaining only one Floquet block. At

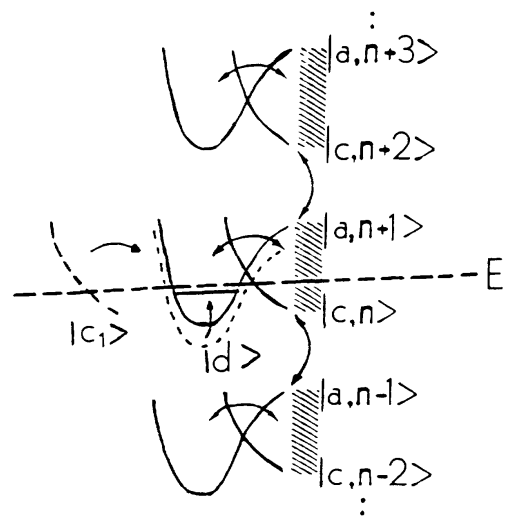

FIG. 1. Schematic representation of the two-artificialchannel method involving the bound $|a\rangle$ and the dissociative $|c\rangle$ electronic states. Each pair of crossing curves (on the right) represents one Floquet block radiatively coupled to two adjacent ones as indicated by the arrows. They are open or closed according to their asymptotic energies as compared to the collision energy $E$ under consideration. $\left|c_{1}\right\rangle$ and $|d\rangle$ are the two asymmetrically coupled artificial channels. 
least three figures of accuracy are obtained and the results are in full agreement with those of Refs. [2] and [3] based on the calculation of resonance widths, showing, in particular, that for such radiative couplings one isolated laser-induced resonance is responsible for the line shape $\sigma(\omega)=\left|T_{c a}\left(E_{a}+\hbar \omega\right)\right|^{2} / I$. The full curve of Fig. 2 represents this weak-field behavior, the different types of calculations leading to results too close to each other to be distinguished at that scale. We also note that at sufficiently low intensities, photodissociation proceeds via the first energetically accessible continuum state which requires absorption of one photon and yields the lowest relative kinetic energy $\epsilon(1)$, which means that $P_{c, a} \simeq 1$.

\section{B. Intermediate-field regime}

For field strengths roughly extending from $I \approx 10^{11}$ $\mathrm{W} / \mathrm{cm}^{2}$ to $I \approx 10^{13} \mathrm{~W} / \mathrm{cm}^{2}$, a nonlinear behavior of the dissociation rates as functions of intensity is obtained. This can be related to off-energy-shell contributions, as well as to neighboring resonance overlaps. A singlephoton description involving one Floquet block but avoiding the use of the perturbative expression, Eq. (18), already contains the required information. Shapiro's one-artificial-channel method with a symmetrical coupling between $|a, n+1\rangle$ and $|c, n\rangle$ leading to an additional shift and width affecting $E_{a}$ in the denominator of Eq. (16) or the more complete formalism of the two-artificialchannel method, Eq. (21), can be used to get the photodissociation line shape which is represented by the triangles of Fig. 2 for $I \simeq 2 \times 10^{12} \mathrm{~W} / \mathrm{cm}^{2}$ (no appreciable difference between the two methods at that scale). In agreement with previous calculations, the increase of the intensity leads to a slight blueshift of the maximum of the line shape as well as a flattening for large wavelengths. It is to be noticed that the unique Floquet block used in these calculations gives a convergence up to $98 \%$ and the difference between the two calculations (one or two artificial channels) is less than $0.6 \%$.

For a given wavelength of the laser, it may be interesting to study the interferences arising from the interaction of higher vibrational $(v=1,2,3, \ldots, j=1)$ field-induced

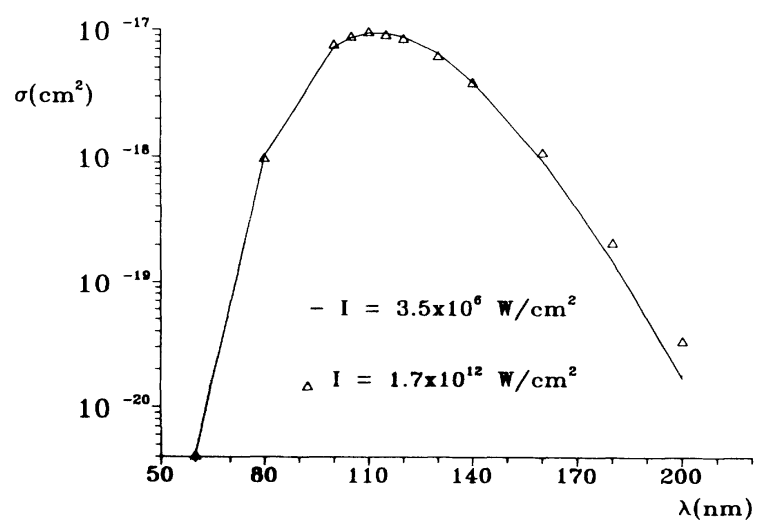

FIG. 2. Photodissociation line shape for the $(v=0, j=1)$ level of $\mathrm{H}_{2}^{+}$ground state, for two laser intensities corresponding to the weak- (solid line) and intermediate-field (triangles) regime. Wavelengths are given in $\mathrm{nm}$. resonances which are supported by the $|a, n+1\rangle$ dressed state coupled to the $|c, n\rangle$ continuum. This can be done by a global energy variation of the Floquet block relative to the fixed position of the artificial channels $\left|c_{1}\right\rangle$ and $|d\rangle$, such as the collision energy $E$ which enters in Eq. (21) scans all quasienergy levels of $|a, n+1\rangle$. The results, for $\lambda=120 \mathrm{~nm}$, with $I=8.8 \times 10^{11} \mathrm{~W} / \mathrm{cm}^{2}$ and $I=3.5 \times 10^{12} \mathrm{~W} / \mathrm{cm}^{2}$, are displayed in Figs. 3(a) and 3(b) as $\left|S_{k c_{1}}\right|^{2}(k$ being an index for the $k$ th open channel; i.e., on Fig. $1, k=1$ for $|c, n\rangle ; k=2$ for $|a, n-1\rangle ; k=3$ for $|c, n-2\rangle$ and so on) on a logarithmic scale, versus energy $E$. For the lowest intensity [Fig. 3(a)], clearly five peaks can be identified which roughly correspond to the positions of $(v=0,1,2,3,4 ; j=1)$ resonances (i.e., the positions of the first five vibrational bound states of $|a\rangle$, affected by a field-induced shift). The successive peak amplitudes are very different (approximately one or two orders of magnitude of decrease between each of them). This is a direct consequence of the preparation step concerning the lowest vibrational level of $|a\rangle$. Via the asymmetric coupling $V_{d a}=0, V_{a d}=1,|d\rangle$ is only coupled to the ( $v=0, j=1$ ) level of $|a\rangle$, such that this level is picked out as the initial state which in turn is distributed over the complex dressed states $|L\rangle$. The resulting line shape presents an important maximum when the $(v=0, j=1)$ level of $|a, n+1\rangle$ is in a doorway situation with respect to $E$ (as is illustrated in Fig. 1). Upper resonances $(v=1,2,3,4 ; j=1)$ weakly interfere with $(v=0, j=1)$, only via their coupling through the continuum $|c, n\rangle$, producing Fano profiles [10] with a peak and a dip structure, but with rather low amplitudes, such as one could hardly distinguish them in a linear scaled graph. When the field strength is varied from $8.8 \times 10^{11} \mathrm{~W} / \mathrm{cm}^{2}$ to $3.5 \times 10^{12} \mathrm{~W} / \mathrm{cm}^{2}$ [Fig. 3(b)] the position of the $(v=0, j=1)$ peak is shifted and its width is increased approximately as a linear function of the intensity. Fano profiles corresponding to the $(v=1,2,3,4, \ldots ; j=1)$ levels, which are indirectly coupled among them via $|c, n\rangle$ and $|a, n+1(v=0, j=1)\rangle$, are also shifted and broadened, their amplitudes remaining however moderate as compared with the dominant peak.

\section{Strong-field regime}

For higher intensities, multiphoton absorption and emission processes are to be considered which are at the origin of marked nonlinearities affecting the line shapes. Parallel to this, depending on the photon energy $\hbar \omega$ and initial state, absorption of additional photons leads to multiphoton dissociation pathways that are much more favorable than the minimum-energy path. We refer to such processes as above-threshold dissociation to emphasize their similarity with the above-thresholdionization process [11]. Several Floquet blocks have to be introduced simultaneously in the calculation (open and closed) and a careful optimization study is to be carried out for the determination of the most efficient blocks to fasten convergency. Typically nine Floquet blocks (four closed and five open) plus the resonant one are necessary to achieve three figures of accuracy. Figures 3(c) and 3(d) display converged results for $\left|S_{k c_{1}}\right|^{2}$ at the same wave- 

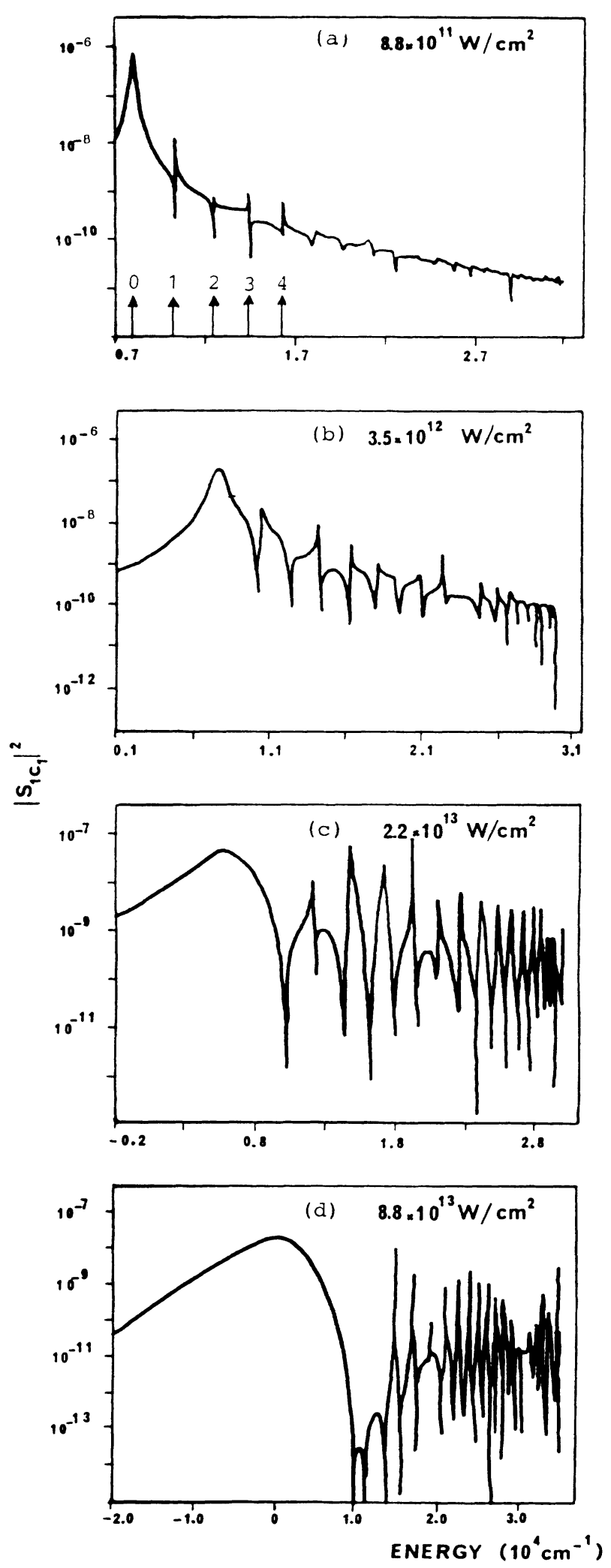

FIG. 3. Square modulus of the scattering matrix element $\left|S_{1 c_{1}}\right|^{2}$ as a function of the collision energy for a laser wavelength $\lambda=120 \mathrm{~nm}$, in (a) weak-, (b) intermediate-, and (c) and (d) strong-field regimes. The arrows in (a) indicate the position of unperturbed vibrational levels of $\mathrm{H}_{2}{ }^{+}\left(1 s \sigma_{g}\right)$. length and for intensities of $2.2 \times 10^{13} \mathrm{~W} / \mathrm{cm}^{2}$ and $8.8 \times 10^{13} \mathrm{~W} / \mathrm{cm}^{2}$ The preparation step again favors the resonance corresponding to the lowest vibrational level of $|a, n+1\rangle$ to which $|d\rangle$ is directly coupled. The resulting peak presents a rather large bandwidth with a highly asymmetric behavior showing marked tendency to flatten at lower energies, in accordance with the situation already depicted in Fig. 2. However, here, the laser induces an avoided crossing so that the $(v=0, j=1)$ level, from the $|a\rangle$ initial state, is severely broadened through tunneling $[2,4]$. With such high intensities the other complex dressed states are strongly interfering with each other. Important shifts and widths are affecting the resonances mediating the dissociation dynamics leading to a complicated peak and dip pattern which cannot be easily correlated with the set of vibrational levels of $|a, n+1\rangle$. The first 18 oscillations seem however to be in relation with the 18 vibrational levels supported by the $|a, n+1\rangle$ channel potential. Due to the strong coupling, they appear to be equally spaced (the molecular anharmonicity is washed out by the field). The additional series of structures appearing at energies above $28000 \mathrm{~cm}^{-1}$ are certainly due to resonances originating from upper Floquet blocks. We have also to note that Fano profiles exhibit amplitudes which are of the same order of magnitude than the leading $(v=0, j=1)$ peak.

Information concerning the branching ratios $\rho_{k a}$ is plotted in Fig. 4 as a function of the laser-field-intensity ranging between $10^{8}-10^{14} \mathrm{~W} / \mathrm{cm}^{2}$. They represent the relative probabilities to dissociate into channels $|c, n\rangle,|a, n-1\rangle$, and $|c, n-2\rangle$. The wavelength which is chosen for the calculations, namely $\lambda=329.7 \mathrm{~nm}$, favors a three-photon transition from $(v=0, j=1)$. Three different intensity regimes are obtained which can be interpreted in an adiabatic picture as follows.

(i) At field strengths below $3.5 \times 10^{12} \mathrm{~W} / \mathrm{cm}^{2}$, the onephoton absorption resulting into fragments in channel $|c, n\rangle$ has a very low probability due to a high and wide tunneling barrier originating from an adiabatic decoupling of the surfaces associated with $|a, n+1\rangle$ and $|c, n\rangle$. The absorption dynamics rather proceeds via a threephoton jump to the $|c, n-2\rangle$ channel. The branching ratio $\rho_{3 a}$ largely dominates over the other dissociation paths.

(ii) At higher intensities, the most striking observation is that, in spite of the $I^{3}$ dependence of the total photodissociation rate, the relative probability to find fragments in channel $|a, n-1\rangle$ dominates over $|c, n-2\rangle$ $\left(\rho_{2 a}>\rho_{3 a}\right)$. Such a situation has already been discussed in the literature from both experimental [12] and theoretical [11] points of view. The avoided curve crossing between channels $|c, n-2\rangle$ and $|a, n-1\rangle$ taking part in the dynamics is invoked as an explanation. In the proximity of this point the three-photon-dissociating system returns one photon to the radiation field via stimulated emission, favoring thus the branching ratio of channel $|a, n-1\rangle$. The adiabatic path leading to $\rho_{2 a}>\rho_{3 a} \gg \rho_{1 a}$ (threephoton absorption, followed by an emission during dissociation resulting into velocity lowering of the protons) appears to be preferred for increasing fields. This is precisely what one gets for intensities ranging between $3.5 \times 10^{12}$ 


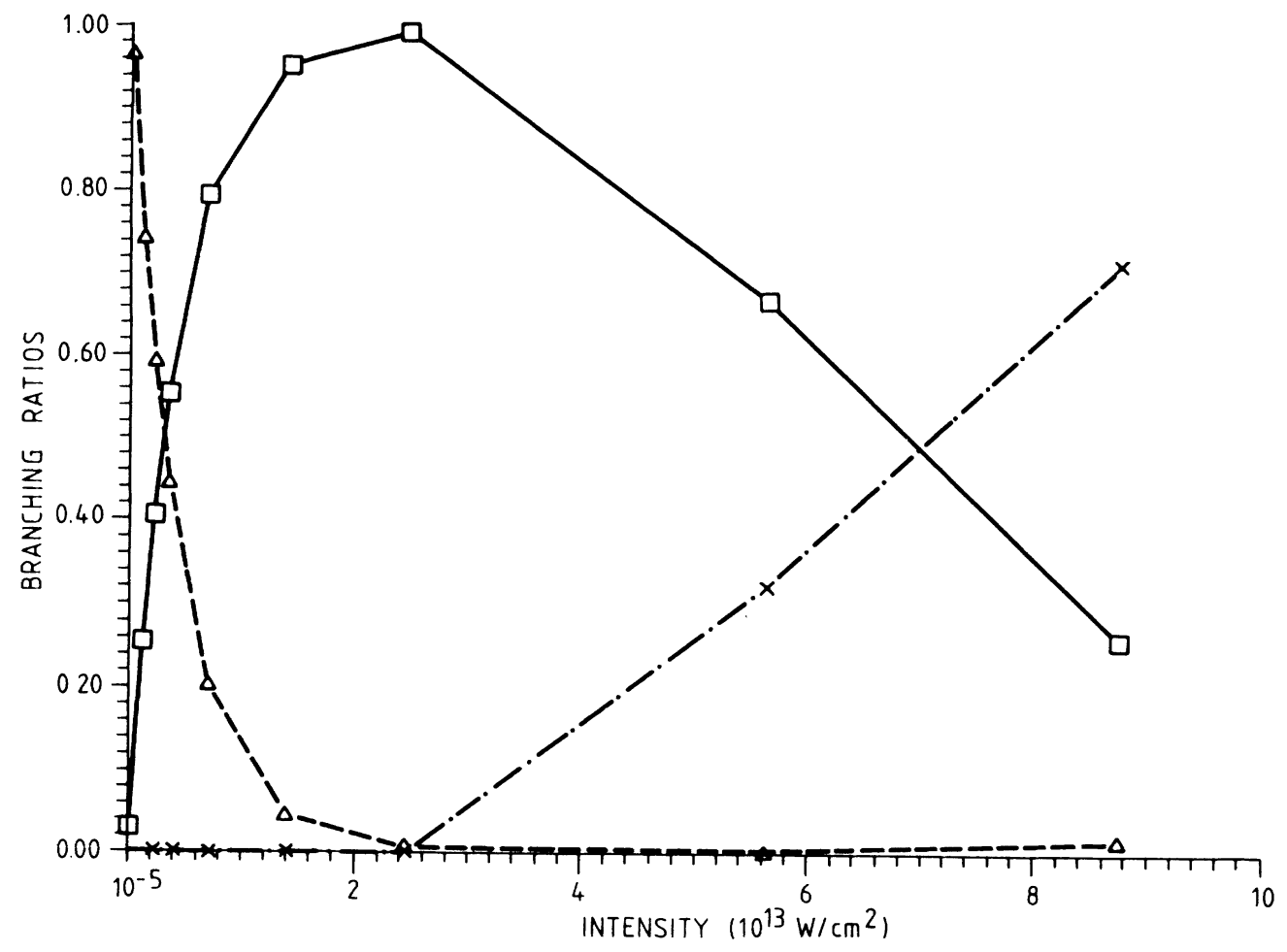

FIG. 4. Branching ratios as a function of the field strength over the range $10^{8}-10^{14} \mathrm{~W} / \mathrm{cm}^{2}$ for a photon wavelength $\lambda=329.7 \mathrm{~nm}$. Crosses denote the one-photon channel $\rho_{1 a}$; squares denote the two-photon channel $\rho_{2 a}$; and triangles denote the three-photon channel $\rho_{3 a}$.

$\mathrm{W} / \mathrm{cm}^{2}$ and $2.5 \times 10^{13} \mathrm{~W} / \mathrm{cm}^{2}$.

(iii) Above $3 \times 10^{13} \mathrm{~W} / \mathrm{cm}^{2}$ the dissociation probability towards channel $|c, n\rangle$ increases to such an extent that for $I=7 \times 10^{13} \mathrm{~W} / \mathrm{cm}^{2}$ it is of the same order as for channel $|a, n-1\rangle$ and for higher values of the field $\left(10^{14}\right.$ $\mathrm{W} / \mathrm{cm}^{2}$ ) it describes the dominant process. A possible interpretation would be the lowering and flattening of the adiabatic potential barrier at the avoided curve crossing occurring between $|a, n+1\rangle$ and $|c, n\rangle$. An estimate of changes that may affect dressed potential energy surfaces by intense radiation fields leading to important tunneling has very recently been given [13]. It appears that for the case of $\mathrm{H}_{2}{ }^{+}$, field intensities of the order of $5 \times 10^{13}$ $\mathrm{W} / \mathrm{cm}^{2}$ are enough to flatten completely the potential barrier resulting from dressed molecular states with an electromagnetic field of wavelength $\lambda=532 \mathrm{~nm}$.

In conclusion, we have shown the ability of the artificial-channel procedure within a close-coupled equation scheme to reproduce dissociation probabilities in intense-radiative-field regimes, even in the case of overlapping laser induced resonances. The most important result concerns $\mathrm{H}_{2}{ }^{+}$above-threshold photodissociation which is the subject of numerous experimental investigations [12]. Relative probabilities to reach fragmentation channels with 3,2 , or 1 photons of wavelength $\lambda=329.7$ $\mathrm{nm}$ are analyzed and interpreted in terms of radiatively dressed adiabatic molecular states as a function of field strength. It is shown, as in previous calculations [11], that although the process is initiated by a three-photon absorption, at intensities larger than $3.5 \times 10^{12} \mathrm{~W} / \mathrm{cm}^{2}$, one photon is reemitted during the dissociation, yielding thus a lowering of the proton kinetic energy. For intensities above $7 \times 10^{13} \mathrm{~W} / \mathrm{cm}^{2}$ two photons can be reemitted such that the asymptotic fragmentation channel corresponds to a net single-photon absorption process, which seems to be consistent with very recent experimental results [14].
[1] M. H. Mittleman, Introduction ot the Theory of Laser. Atom Interactions (Plenum, New York, 1982).

[2] A. D. Bandrauk and M. L. Sink, J. Chem. Phys. 74, 1110 (1981).

[3] (a) X. He, O. Atabek and A. Giusti-Suzor, Phys. Rev. A 38, 5586 (1988); (b) 42, 1585 (1990).

[4] (a) A. D. Bandrauk and G. Turcotte, J. Chem. Phys. 89, 3039 (1985); (b) A. D. Bandrauk and O. Atabek, in Lasers,
Molecules and Methods, edited by J. O. Hirschfelder, R. E. Wyatt, and R. D. Coalson (Wiley, New York, 1989), Chap. 19.

[5] M. Shapiro, J. Chem. Phys. 56, 2582 (1972); G. G. BalintKurti and M. Shapiro, in Photodissociation and Photoionization, edited by K. P. Lawley (Wiley, New York, 1985).

[6] C. Cornaggia, D. Normand, J. Morellec, G. Mainfray, and C. Manus, Phys. Rev. A 34, 207 (1986); T. S. Luk and C. 
Rhodes, ibid. 38, 6180 (1988); J. Verschuur, L. Noordam, and H. van Linden van den Heuvell, ibid. 40, 4383 (1989); A. Zavriyev, P. H. Bucksbaum, H. G. Muller, and D. W. Schumacher, ibid. 42, 5500 (1990); B. Yang, M. Saeed, L. F. Di Mauro, A. Zavriyev and P. H. Bucksbaum (unpublished).

[7] M. L. Goldberger and K. M. Watson, Collision Theory (Wiley, New York, 1964), Chap. 8.

[8] A. D. Bandrauk, G. Turcotte, and R. Lefebvre, J. Chem. Phys. 76, 225 (1982).
[9] M. Shapiro and H. Bony, J. Chem. Phys. 83, 1588 (1985).

[10] U. Fano, Phys. Rev. 124, 1866 (1961).

[11] A. Giusti-Suzor, X. He, O. Atabek, and F. H. Mies, Phys. Rev. Lett. 64, 515 (1990).

[12] P. H. Bucksbaum, H. G. Muller, D. W. Schumacher, and A. Zavriyev, Phys. Rev. Lett. 64, 1883 (1990).

[13] A. D. Bandrauk, E. Constant, and J. M. Gauthier, J. Phys. II 1, 1033 (1991).

[14] L. F. Di Mauro and P. H. Bucksbaum (private communcation). 